\title{
Intervenção em habilidades sociais com uma criança com síndrome de down
}

\author{
Olga Maria Piazentin Rolim Rodrigues* \\ Larissa Helena Zani dos Santos** \\ Fabiana Cristina Carlino ${ }^{* * *}$
}

\section{Resumo}

Estudos apontam que crianças com Síndrome de Down apresentam déficits em habilidades sociais, o que pode interferir nas suas interações sociais e, também, no seu desempenho acadêmico. O objetivo deste estudo foi analisar a significância clínica e mudanças confiáveis no repertório de habilidades sociais de uma criança com Síndrome de Down, a partir de intervenções na clínica, em casa e na escola. Participaram deste estudo uma menina de sete anos com Síndrome de Down, que freqüentava o primeiro ano do ensino regular, seus pais e sua professora. Eles eram atendidos em um Centro de Psicologia Aplicada, de uma universidade pública do interior paulista. Antes e depois da intervenção, pais e professora responderam ao Sistema de Avaliação de Habilidades Sociais (SSRS). A intervenção com a criança foi realizada durante seis meses, por meio de atividades lúdicas em reuniões semanais. Com os pais e professora, em encontros quinzenais e mensais, foram discutidos diversos temas relacionados às habilidades sociais. Os resultados obtidos foram analisados utilizando o método JT. Com os pais, comparando as duas avaliações conduzidas, os dados apontaram para mudança positiva confiável para quatro das seis habilidades avaliadas. Das cinco habilidades avaliadas pela professora, uma delas não estava no nível clínico desde a primeira aplicação. Das demais, duas passaram de nível clinico para não clínico, caracterizando uma mudança positiva confiável. O estudo mostrou a importância de avaliações pré e pós em estudo de caso, possibilitando a avaliação dos efeitos da intervenção realizada sobre os diversos fatores das habilidades sociais. Estudos futuros com populações maiores poderão referendar os dados obtidos.

Palavras-chave: Intervenção com pais e professores; Habilidades Sociais; Síndrome de Down.

\footnotetext{
* Professora Doutora do Departamento de Psicologia e do Programa de Pós-graduação em Psicologia do Desenvolvimento e Aprendizagem da Faculdade de Ciências, campus de Bauru, da UNESP. Bauru, São Paulo, Brasil.

** Doutoranda do Programa de Pós Graduação em Psicologia da Universidade Federal de São Carlos (UFSCar). São Carlos, São Paulo, Brasil.

*** Professora Doutora do Núcleo de Fonoaudiologia da Universidade Federal de Sergipe (UFS), Campus Lagarto. Lagarto, Sergipe, Brasil.
} 


\section{Intervention in social skills with a child with Down's Syndrome}

\section{Abstract}

Studies show that children with Down's syndrome have deficits in social skills, which can interfere in their social interactions and also in their academic performance. The aim of this study was to analyze the clinical significance and reliable change in repertory of social skills of a child with Down's syndrome, from interventions in the clinic, at home and at school. A seven year old girl with Down's Syndrome who had frequented the first year of regular school, her parents and her teacher participated in this study. They were attending in a Center of Applied Psychology, of a public university in São Paulo State. Before and after the intervention parents and teacher answered the Rating System Social Skills (SSRS). The intervention with the child had been conducted for six months, through playful activities in weekly meetings. It was discussed various topics related to social skills with parents and teacher in biweekly and monthly meetings. The results were analyzed using JT method. With parents, comparing the two assessments conducted, the data pointed to positive change reliable for four of the six skills assessed. Of the five skills assessed by the teacher, one of them was not in the clinical level since the first application. The other two went from clinical to non-clinical level, featuring reliable positive change. The study showed the importance of pre and post measures in case study, enabling the assessment of the effects of the intervention performed on the various factors of social skills. Further studies with larger populations may ratify the data obtained.

Keywords: Intervention; Social Skills; Down's syndrome.

\section{Introdução}

Crianças com síndrome de Down apresentam limitações físicas, intelectuais e sociais, as quais podem ser melhoradas por meio de intervenções eficazes e precoces, uma vez que a exposição direta e assistemática aos estímulos sociais nem sempre é suficiente para promover a aquisição de comportamentos sociais adequados (GAUDERER, 1997).

Para Omote (1990), o fato de pessoas com síndrome de Down apresentarem características físicas facilmente identificáveis tende a ser um dificultador no estabelecimento de interações sociais satisfatórias que acarretam déficits em habilidades sociais. Tais déficits podem se expressar, principalmente, em relação a sociabilidade e aptidões sociais, como cooperação interpessoal e adequação a convenções sociais (ANGÉLICO; DEL PRETTE, 2011). Os déficits em habilidades sociais das crianças com síndrome de Down podem persistir durante a adolescência, resultando efeitos potencialmente negativos em sua adaptação à vida adulta e integração social em ambientes mais amplos (SORESI; NOTA, 2000). 
Habilidades sociais são comportamentos específicos que resultam em interações positivas e abrangem tanto comportamentos verbais, quanto não-verbais, necessários para uma comunicação interpessoal efetiva (GRESHAM, 1986). O termo Habilidades Sociais tem sido definido como a atuação do indivíduo diante de uma situação interpessoal (DEL PRETTE; DEL PRETTE, 1999). São "comportamentos aprendidos e socialmente aceitáveis que permitem ao individuo interagir efetivamente com os outros e evitar ou fugir de comportamentos não aceitáveis que resultem em interações sociais negativas" (GRESHAM 2009, pg. 19). O autor ressalta que tais habilidades, se presentes no repertório de crianças e adolescentes, facilitam a iniciação e manutenção de relações sociais positivas, contribuindo para a aceitação de colegas, resultando, principalmente, em ajustamento escolar satisfatório.

Beidel, Turner e Morris (2000) descrevem exemplos de habilidades sociais que incluem: sorrir e fazer contato olho a olho, perguntar e responder, dar e reconhecer elogios durante uma troca social. Os autores também discutem que essas habilidades são aprendidas e o seu desempenho está relacionado ao estágio de desenvolvimento do indivíduo. As habilidades dependem das variáveis ambientais e cognitiva e, ainda, da interação entre esses aspectos. Para Del Prette e Del Prette (2001) as habilidades sociais contribuem para o desenvolvimento sadio do indivíduo.

Del Prette e Del Prette (2005) ressaltam que déficits em habilidades sociais podem se expressar sob três formas: na aquisição, quando a habilidade não ocorre; no desempenho, quando a habilidade é apresentada em uma freqüência inferior a esperada e, na fluência, quando a habilidade é apresentada com proficiência inferior a esperada. Segundo os autores, alguns fatores pessoais e ambientais podem estar relacionados aos déficits de habilidades sociais da criança, como: falta de conhecimento, restrição de oportunidade e modelo, problemas de comportamento, ausência de feedback, falhas de reforçamento, ansiedade interpessoal excessiva e dificuldade de discriminação e processamento.

Mancini, Silva, Gonçalves e Martins (2003) realizaram um estudo com um grupo de crianças com síndrome de Down e um grupo de crianças com desenvolvimento típico, com objetivo de comparar o desempenho funcional (autocuidado, mobilidade e função social) dessas crianças utilizando o teste funcional PEDI. Nos achados, os autores identificaram atraso no grupo de crianças com síndrome de Down no desempenho de atividades que envolviam a comunicação expressiva, compreensão, socialização e resolução de problemas.

Bonomo, Garcia e Rosseti (2009) realizaram uma pesquisa com o objetivo de apresentar uma descrição inicial dos relacionamentos interpessoais de 10 adolescentes com síndrome de Down e seus responsáveis, enfatizando suas amizades, por meio de entrevistas semiestruturadas. Os dados obtidos apontaram para o fato de que esses adolescentes possuem amigos, mas é uma amizade caracterizada por atitudes de ajuda e de companheirismo em atividades praticadas por ambos. Atividades extracurriculares foram pouco relatadas, indicando um quadro de exclusão quando comparados com adolescentes com desenvolvimento típico. As análises mostraram que as amizades são qualitativamente diferentes das observadas em adolescentes 
com desenvolvimento típico. Os autores ressaltam que: compreender esses tipos de limitações contribui com o desenvolvimento de programas que estimulem adolescentes com síndrome de Down e seus companheiros fortalecerem seus vínculos.

Beltrame, Herber e Ribeiro (2009) realizaram um estudo com objetivo de analisar a socialização de uma criança com síndrome de Down matriculada em uma escola regular. Foi realizada uma pesquisa descritiva-qualitativa, do tipo estudo de caso. Foram participantes da pesquisa a professora e 20 alunos do $3^{\circ}$ ano, sendo um deles com síndrome de Down. Como instrumento de coleta de dados, foram utilizados uma entrevista semi-estruturada e um teste sociométrico. A partir da avaliação do teste sociométrico pode-se constatar que o sujeito com síndrome de Down foi escolhido como primeira escolha lúdica apenas por um colega, segundo relato da professora, classificando-se na linha média da sociometria enquanto que, na segunda escolha lúdica e escolhas afetivas, o sujeito encontrava-se no limite inferior. Concluiu-se que o participante do estudo encontra-se envolvido em díades o que levou os autores a inferirem que o processo inclusivo favorece a convivência em comunidade e a inserção social de pessoas com síndrome de Down.

Lucisano, Pfeifer, Panuncio-Pinto, Anhão e Santos (2011) realizaram um estudo com o objetivo de identificar as habilidades sociais de crianças com síndrome de Down no processo de interação na rede regular de educação infantil, de um município no interior do estado de São Paulo. Participaram dessa pesquisa seis crianças com síndrome de Down na faixa etária de cinco e seis anos. Foram analisadas quatro filmagens de cada criança em situações de interação social na sala de aula e nas atividades extracurriculares, considerando suas habilidades interpessoais e de auto-expressão. Quanto às habilidades interpessoais, nos comportamentos "Ocorre interação com outra criança", "Ocorre interação com educadora", "Ocorre interação com objeto" e "Brinca junto, com o mesmo tipo de objeto" apresentaram frequência elevada em todas as crianças. Quanto às habilidades de autoexpressão, o comportamento "Sorrir" apresentou maior frequência, presente em todas as crianças avaliadas. O comportamento "Imita outras crianças" foi observado em todas as crianças avaliadas durante as atividades extracurriculares. Os autores concluem, sobre a importância da inclusão na rede regular, que facilitam a aquisição de repertório social adequado para a vida em sociedade.

Angélico e Del Prette (2011) utilizou um método observacional para avaliar o repertório social de 10 adolescentes com síndrome de Down. Para o levantamento das habilidades sociais, constantes no repertório de interação social dos sujeitos, foram efetuadas sete sessões de filmagens para cada um dos participantes em diversas situações naturais na escola e duas em situações estruturadas. Para análise das filmagens foram estabelecidas quatro categorias de habilidades pró-sociais: comunicativas, civilidade, empáticas e de expressão de sentimento positivo, além de uma classe de habilidades de enfrentamento. Todos os participantes demonstraram ter em comum no seu repertório as seguintes habilidades sociais: iniciar contato e conversação, estabelecer contato visual, fazer perguntas, responder perguntas, concordar (ação verbal), acatar ordens, prestar atenção e sorrir para o outro. Os resultados mostraram que a maioria apresentou déficits de respostas assertivas de enfrentamento em seu re- 
pertório comportamental, prevalecendo respostas passivas,o que poderia ser indicativo de necessidade de intervenções preventivas e educacionais que assegurem a eles um melhor desempenho em situações de demandas para situações de enfrentamento.

Pereira (2007) realizou um estudo com os objetivos de identificar o repertório de habilidades sociais de estudantes com síndrome de Down e o repertório de habilidades sociais de estudantes com desenvolvimento típico, comparar o repertório desses dois grupos, comparar auto avaliação e avaliação dos professores. Participaram 10 crianças com síndrome de Down, incluídas em classes de escolas regulares de ensino, e 10 crianças com desenvolvimento típico. Para atingir seus objetivos, a pesquisadora utilizou o Inventário Multimídia de Habilidades Sociais de Crianças (IMHSC - Del Prette). Os resultados da pesquisa apontaram que as crianças com síndrome de Down, no geral, demonstraram ter um repertório de habilidades sociais deficitário. Foi identificada, também, correlação positiva entre auto avaliação e avaliação dos professores. O grupo de crianças com síndrome de Down variou no nível de habilidades sociais de acordo com a escola e dificuldade de aprendizagem.

Anhão, Pfeifer e Santos (2010) compararam a interação social de seis crianças com síndrome de Down e seis crianças com desenvolvimento típico na faixa etária de três a seis anos, da rede regular de educação infantil. Cada criança foi analisada através de categorias que envolvem o processo de interação social em ambiente escolar. Os resultados mostraram que as crianças com síndrome de Down, quando comparadas às crianças com desenvolvimento típico, revelaram déficits nas habilidades sociais assertivas, ou seja, elas dependem de maior iniciativa de outros e, como consequência, apresentaram índice mais alto de habilidades sociais passivas.

Na revisão de estudos com crianças síndrome de Down e deficiência intelectual, Freitas (2011) demonstrou que essas crianças apresentam: limitações no repertório de habilidades sociais gerais e específicas; limitações em competência social; indicadores de status sociométrico negativo; problemas de comportamento gerais, internalizantes e externalizantes; passam a maior parte do recreio sozinhos e apresentam déficits em comportamentos adaptativos.

Segundo Teixeira e Kubo (2008), a produção de conhecimento sobre as interações sociais de crianças com síndrome de Down, constitui uma necessidade científica e social, uma vez que pode oferecer subsídios para a caracterização e aperfeiçoamento de intervenções adequadas, contribuindo, principalmente, nos processos de inclusão escolar. Anhão, Pfeifer e Santos (2010) também ressaltam a importância da realização de estudos que contribuam efetivamente no campo das habilidades sociais de crianças com síndrome de Down, considerando que os avanços teóricos são importantes para a fundamentação de práticas educativas e interventivas, a fim de melhorar o desenvolvimento e a qualidade de vida dos envolvidos. Angélico e Del Prette (2011) também discutem a escassez de estudo, nessa temática, e relevância de pesquisas nessa área. 
Os déficits em habilidades sociais identificados nos estudos relatados com crianças com síndrome de Down parecem interferir, não somente nas interações sociais dessas crianças, mas também no seu desempenho acadêmico, podendo resultar em problemas de comportamento (PEREIRA, 2007; ANHÃO; PFEIFER; SANTOS, 2010; TEIXEIRA; KUBO, 2008). Tais constatações indicam a necessidade de intervenções pontuais, focalizando as habilidades sociais de crianças com síndrome de Down, tendo em vista a carência de pesquisas na área.

Anhão, Pfeifer e Santos (2010) ressaltam a necessidade de estudo que investiguem as diversas classes de comportamentos sociais, que contribuam mais efetivamente no campo das habilidades sociais, no qual os avanços teóricos são importantes para a fundamentação de práticas educativas, subsidiando ações interventivas a fim de melhorar o desenvolvimento e a qualidade de vida dos envolvidos. Considerando tais aspectos, o objetivo desse estudo foi analisar os efeitos de uma intervenção em habilidades sociais com uma criança com síndrome de Down.

\section{Método}

\section{Cuidados Éticos}

O projeto desse estudo foi submetido ao Comitê de Ética em Pesquisa com Seres Humanos da Universidade Estadual Paulista Júlio de Mesquita, recebendo aprovação (Processo nº 926/46/01/11).

\section{Participantes}

Participaram desse estudo uma criança do sexo feminino com Síndrome de Down, de sete anos, que frequentava a primeira série do ensino regular, seus pais e sua professora. A criança era atendida em um Centro de Psicologia Aplicada, de uma universidade pública do interior paulista.

\section{Materiais}

Para avaliar o repertório de Habilidades Sociais da criança foi utilizado o instrumento Sistema de Avaliação de Habilidades Sociais. A validação desse instrumento pode ser consultada no artigo de Bandeira, Del Prette, Del Prette e Magalhães (2009). O instrumento conta com três escalas de avaliações: habilidades sociais, problema de comportamento e desempenho acadêmico, para este estudo foi utilizada apenas a escala de habilidades sociais. O instrumento também possui três versões: para auto avaliação do aluno, para pais e para professores. Para este estudo foram utilizados somente os formulários que avaliam as habilidades sociais para pais e professores.

Os pais respondem a 38 itens que avaliam a frequência e a importância das habilidades sociais da criança, distribuídos nas categorias: Fator 1- Cooperação (Comportamentos da criança de colaborar, sem ser solicitado, em tarefas domésti- 
cas, manter o próprio quarto arrumado, guardar os brinquedos etc.); Fator 2- Asserção Positiva (Comportamentos da criança que geram a estima dos demais, como aceitar ideias, pedir permissão, fazer e aceitar elogios.); Fator 3- Iniciativa/Desenvoltura Social (Comportamentos apropriados de iniciar e manter interações sociais para conversar, apresentar-se, fazer amigos, convites, pedir informações, juntar-se a grupos etc.); Fator 4-, Asserção de Enfrentamento (Comportamentos que expressam confiança em lidar com estranhos e situações novas, questionar regras consideradas injustas, relatar acidentes, pedir ajuda etc.); Fator 5-Civilidade (Comportamentos que demonstram domínio sobre as próprias emoções por meio de reações abertas tais como: usar "apropriadamente" o tempo livre, responder "educadamente" a provocações e pedidos abusivos, usar "de modo aceitável" o tempo livre, falar "em tom de voz apropriado" etc.) e, Fator 6- Autocontrole (Comportamentos que demonstram domínio sobre as próprias emoções, por meio principalmente de reações encobertas, tais como: autocontrole de irritação ou raiva em situações de discussão, conflito, críticas, discordâncias etc.)

O professor responde para 30 itens que medem a frequência de emissão de habilidades sociais, nas categorias: Fator l- Responsabilidade/Cooperação (Comportamentos que demonstram compromisso com as tarefas e com as pessoas e disponibilidade da criança para colaborar com o bom andamento das atividades, por exemplo: seguir instruçōes do professor, manter a carteira limpa e arrumada, oferecer-se para ajudar colegas nas tarefas de classe, mostrar interesse nas atividades.); Fator 2- Asserção (Comportamentos que envolvem expor-se e buscar relações com os demais, por exemplo: iniciar conversação com colegas, convidá-los para juntar-se em atividades, apresentar-se a novas pessoas, fazer amigos, juntar-se a grupos, falar positivamente de si, questionar regras que considera como injustas.); Fator 3- Autocontrole (Comportamentos que demonstram domínio das próprias reações emocionais, por exemplo: reagir de forma apropriada à pressão, gozação ou provocação dos colegas, controlar irritação em situações de conflito com colegas, aceitar ideias dos colegas para atividades, negociar em situações de conflito, usar tempo livro de forma aceitável.); Fator 4- Autodefesa (Comportamentos que envolvem enfrentamento para defesa de ideias, opiniões ou avaliações com algum risco de reação indesejável do outro, por exemplo: argumentar para defender-se quando tratado injustamente, questionar regras que considera como injustas, falar coisas boas de si mesmo quanto pertinente.) e, Fator 5- Cooperação com pares (Comportamentos que expressam disponibilidade da criança para colaborar, por exemplo: juntar-se a um grupo ou atividade, ajudar colegas nas tarefas, cooperar voluntariamente, ignorar distrações.).

\section{Procedimento de Coleta de dados}

A terapeuta responsável pela intervenção com a criança no centro de Psicologia e pelo trabalho junto aos pais e professora aplicou, antes do inicio da mesma, as escalas de avaliação de habilidades sociais do referido SSRS com os pais e a professora da criança. Após a avaliação, foi iniciada a etapa de intervenção que constou de 26 sessões de atendimento terapêutico com a criança, 16 sessões com os pais e nove sessões com a professora. As sessões com a criança e com os pais foram realizadas em uma sala de atendimento do centro de psicologia e as sessões com a professora ocorreram na escola da criança. 
Os objetivos da intervenção com a criança nas sessões terapêuticas foram: treino em pedir permissão para realizar tarefas de sua preferência; pedir desculpas sem ser lembrada e cumprir regras estabelecidas durante as sessões. Para criar condições para emissão de comportamentos adequados, durante os atendimentos, eram estabelecidas regras de como comportar-se a cada atividade, acadêmicas ou lúdicas, impondo limites e ensinando-a a realizar interações com outras pessoas presentes naquele contexto. As intervenções com a criança foram realizadas por meio de atividades lúdicas temáticas (festa junina, Olimpíadas, Folclore, aniversário da criança, dia das crianças e independência do Brasil, entre outras), leitura de livros, atividades de recortar e colar, de pintura, uso de jogos, como memória, quebra-cabeças e de encaixe, atividades com massinha, com jogos criativos como: pequeno engenheiro, lego e fazendinha, atividades com música e com fantoches. Em todas as sessões eram descritas as regras para a permanência na sala. A cada sessão eram apresentadas as atividades com materiais de escolha da terapeuta ou da criança e eram, então, definidas as regras para o seu uso. Caso as regras não fossem cumpridas a sessão seria encerrada. Oportunidades eram criadas para a solicitação de materiais na secretaria, para a escolha de brinquedos na sala dos brinquedos e a participação em brincadeiras com outros usuários do Centro na brinquedoteca. Como tais ocasiões envolviam uma gama maior de comportamentos sociais, alguns eram treinados antes da sua ocorrência (como solicitar copo para água à secretária) ou eram ensinados em situações naturais (como solicitar para participar da brincadeira de outras crianças, quando na brinquedoteca).

Os objetivos da intervenção com os pais foram: identificar e treinar habilidades parentais; esclarecer importância das regras e limites em casa, estabelecendo com eles uma rotina familiar propícia para a ocorrência e cumprimento das regras estabelecidas; ensinar a substituir os comportamentos inadequados por habilidosos e possibilitar a independência da filha, criando condições para que ela exerça com responsabilidade e compromisso as atividades. Nas sessões com os pais foram discutidos temas relacionados aos comportamentos inadequados e aos adequados esperados de seus filhos para, assim, propor procedimentos que possam ser colocados em prática nas situações do cotidiano. O objetivo era ensiná-los a extinguir os comportamentos inadequados e aumentar a frequência dos comportamentos adequados, reforçando -os. Textos sobre educação dos filhos, focando o tema sobre regras e limites foram lidos e discutidos com o casal a fim de propiciar práticas parentais mais eficientes. Entre os temas, estavam textos sobre a importância de se organizar uma rotina para os membros da casa, visando a melhoria das condições de vida da criança e de seus familiares, aumentando a previsibilidade dos acontecimentos rotineiros. Também, foram investigados durante as sessões comportamentos inadequados apresentados pela criança em casa e na escola, o que possibilitou a análise funcional subsidiando ações que poderiam resultar na sua alteração.

Os objetivos da intervenção com a professora foram: trabalhar as relações que levam aos comportamentos inadequados dos alunos; orientar quais alterações no comportamento dela poderiam surtir modificações nos comportamentos das crianças; orientar a professora no sentido de reforçar comportamentos de realização das atividades escolares e, auxiliar a professora no estabelecimento de regras eficientes e na consequenciação apropriada dos comportamentos adequados/inadequados dos alunos. $\mathrm{Na}$ intervenção com a professora, a partir de observações realizada na sala de 
aula, foram discutidas alternativas para extinção de comportamentos inadequados dos alunos e para o reforço dos comportamentos adequados. Durante as visitas, foram investigados os comportamentos da criança nas semanas anteriores da visita e, após esse procedimento, era discutido com a professora formas de alterá-los com vistas a expansão do repertório de habilidades sociais no contexto escolar.

Ao final da intervenção, a terapeuta aplicou novamente as escalas de habilidades sociais com os pais e a professora.

\section{Análise dos dados}

Os dados obtidos com o instrumento SSRS (BANDEIRA et al., 2009) nas duas aplicações (pré e pós) foram submetidos a tratamento estatístico do Programa SPSS for Windows 17.0, para o cálculo dos Escores Fatoriais.

Para a versão dos pais, foram calculados desempenhos em cada uma das categorias: Fator 1- Cooperação; Fator 2- Asserção Positiva; Fator 3- Iniciativa/Desenvoltura Social; Fator 4- Asserção de Enfrentamento; Fator 5- Civilidade e, Fator 6- Autocontrole. Para a versão do professor, foram calculados: Fator 1- Responsabilidade/Cooperação; Fator 2- Asserção; Fator 3- Autocontrole; Fator 4- Autodefesa e, Fator 5-Cooperação com pares.

O cálculo de cada fator pelo programa SPSS fornece valores que podem ser consultados pela tabela normativa e possibilita a consulta dos percentuais e classificação de acordo com a amostra normativa (ver BANDEIRA et al., 2009). Posteriormente, esses dados foram analisados, por meio do método JT (JACOBSON; TRUAX, 1991; 1992) que possibilita verificar se houve mudança positiva confiável e, também, a significância clínica, utilizando o Programa Excel 2003. Segundo Del Prette e Del Prette (2008), as evidências da efetividade de qualquer intervenção psicológica baseiam-se na demonstração de sua validade interna e externa, usualmente efetuadas por meio de estatística inferencial. Os autores discutem que uma alternativa, mais recentemente explorada, especialmente no âmbito da pesquisa em psicoterapia, é a demonstração da confiabilidade e significância clínica das mudanças, dentre os métodos utilizados na produção desses indicadores, destaca-se o desenvolvido por Jacobson e Truax (1991) mais conhecido como "Método JT". Quando os métodos tradicionais de análise estatística não são aplicáveis (por problemas de amostragem ou de dificuldade de replicação), torna-se necessário investir no desenvolvimento de métodos alternativos (AGUIAR; DEL PRETTE; AGUIAR; DEL PRETTE, 2010).

Jacobson e Truax (1991; 1992) propuseram um método estatístico de tratamento de dados (Método JT) para analisar a confiabilidade das mudanças entre os escores pré e pós-intervenção (Îndice de Mudança Confiável - IMC) e a significância clínica de tais mudanças. Neste método, o IMC serve para determinar se as mudanças entre a pré e a pós-intervenção verificadas para cada indivíduo ou para a média do grupo (mesmo sem grupo controle) que se devem aos procedimentos utilizados ou se constituem artefatos ou erros de medida. Já a análise da significância clínica permite 
verificar o grau em que clientes atingiram os padrões esperados de melhora ou de recuperação de saúde (JACOBSON; TRUAX, 1992).

A significância clínica menos arbitrária seria aquela em que "o nível de funcionamento após a terapia coloca o cliente mais perto da média da população 'normal' do que da média da população disfuncional" (JACOBSON; TRUAX, 1991).

Os autores propõem uma fórmula para se verificar, inicialmente, se as eventuais oscilações positivas ou negativas nos indicadores quantitativos podem ser tomadas confiavelmente como mudanças, definindo o índice de mudança confiável (IMC) como a diferença entre o resultado da pós-intervenção e o da pré-intervenção ponderado pelo índice de confiabilidade do instrumento utilizado na avaliação. Jacobson e Truax (1992) alertam, no entanto, que o IMC, por si só, tem pouco a ver com a significância clínica. Para a significância clínica, é necessário considerar se as mudanças nos escores de uma pessoa que passou por tratamento foram suficientemente robustas para incluí-la na distribuição normativa de resultados da população não clínica.

A expectativa de Jacobson e Truax (1992), com essa metodologia, era a de definir um critério de mudança que pudesse, pelo menos teoricamente, ser aplicável a qualquer desordem clínica e que fosse consistente com as expectativas dos profissionais sobre os resultados desejáveis em psicoterapia. De acordo com Margison et al. (2000), o Método JT é útil tanto para informar progressos de um grupo sob intervenção, quanto para avaliar os progressos de cada indivíduo desse grupo.

A análise do índice de mudança confiável (IMC) possibilita três tipos de classificações: mudança positiva confiável (IMC)1,96), que se localiza acima do intervalo de confiança diagonal; mudança negativa confiável (IMC<-1,96), que se localiza abaixo do intervalo de confiança diagonal e, ausência de mudança confiável, que se localiza dentro ou exatamente sobre o intervalo de confiança diagonal. A significância clínica mede mudanças obtidas que equiparam o indivíduo à população funcional (mudança de status clínico), a partir de dois tipos de possibilidades de resultados: mudança clinicamente significante, podendo gerar dados da população clínica para a não clínica, que se localiza acima do intervalo horizontal e, da passagem da população não clínica para a clínica, que se localiza abaixo do intervalo horizontal. A ausência de significância clínica se localiza dentro ou exatamente em cima do intervalo de confiança horizontal.

\section{Resultados}

Os dados de pais e professores da criança serão apresentados a seguir em tabelas que mostram as análises da mudança confiável e da significância clínica das medidas de pré e pós intervenção de cada uma das categorias elegidas para cada um dos participantes (pais e professora). 
Os resultados gerais da avaliação dos pais do SSRS-BR mostram que, da primeira para a segunda aplicação, houve mudança positiva confiável, com IMC igual a 4 e significância clínica, já que mudou de população clínica para não clínica, passando de um escore pré 26 para um escore pós de 48 , acima do escore médio $(34,23)$ da população não clínica. Dados detalhados de cada um dos fatores estão apresentados na Tabela l. Ela apresenta os dados obtidos com as análises dos fatores das habilidades sociais avaliadas com o SSRS-BR, junto aos pais nas duas aplicações conduzidas, antes e depois da intervenção. Os resultados obtidos mostram que para o Fator l, Cooperação e para o Fator 6, Autocontrole, ocorreu ausência de mudança já que o IMC foi igual à 1 , com nível de significância clínica nas duas aplicações realizadas. Para o Fator 2, Asserção Positiva (IMC=2), o Fator 3, Iniciativa e Desenvoltura Social (IMC=4), o Fator 4, Asserção de Enfrentamento (IMC=4) e o Fator 5, Civilidade (IMC=3), além da mudança positiva confiável, ocorreu mudança clinicamente significativa, já que a criança pertencia anteriormente à intervenção, nestes fatores, ao escore referente à população clínica, alterando para o escore referente a população não clínica após a intervenção.

Tabela 1. Descrição dos resultados obtidos com os pais antes e depois da intervenção.

\begin{tabular}{|c|c|c|c|c|c|}
\hline \multirow[t]{2}{*}{ № } & \multirow[t]{2}{*}{ Fatores } & \multicolumn{2}{|c|}{ Status Clínico } & \multicolumn{2}{|c|}{$\begin{array}{c}\text { Índice de Mudança } \\
\text { Confiável }\end{array}$} \\
\hline & & $1^{\underline{a}}$ Aplicação & 2ª Aplicação & IMC & Classificação \\
\hline 01 & Cooperação & Clínico & Clínico & 1 & $\begin{array}{l}\text { Ausência de } \\
\text { Mudança }\end{array}$ \\
\hline 02 & Asserção Positiva & Clínico & Não clínico & 2 & $\begin{array}{c}\text { Mudança Positiva } \\
\text { Confiável }\end{array}$ \\
\hline 03 & $\begin{array}{l}\text { Iniciativa e desen- } \\
\text { voltura social }\end{array}$ & Clínico & Não clínico & 4 & $\begin{array}{c}\text { Mudança positiva } \\
\text { Confiável }\end{array}$ \\
\hline 04 & $\begin{array}{l}\text { Asserção e En- } \\
\text { frentamento }\end{array}$ & Clínico & Não clínico & 4 & $\begin{array}{c}\text { Mudança Positiva } \\
\text { Confiável }\end{array}$ \\
\hline 05 & Civilidade & Clínico & Não clínico & 3 & $\begin{array}{c}\text { Mudança Positiva } \\
\text { Confiável }\end{array}$ \\
\hline 06 & Autocontrole & Clínico & Clínico & 1 & $\begin{array}{l}\text { Ausência de } \\
\text { Mudança }\end{array}$ \\
\hline
\end{tabular}

Os dados gerais obtidos a partir do relato da professora também apontou para mudança positiva confiável, com IMC igual a 2, porém, não houve significância clínica, pois já pertencia à população não clínica já na primeira aplicação, passando de um escore 38 para o 45, sendo que a média da população não clínica é 33,74. Todavia, análises de cada um dos fatores possibilitam um visão mais acurada dos resultados obtidos depois da intervenção.

Na Tabela 2, estão apresentados os dados obtidos com a professora, dos fatores específicos às habilidades sociais. $\mathrm{Na}$ análise do Fator $\mathrm{l}$, Responsabilidade e Cooperação, ocorreu ausência de mudança já que o IMC foi igual à l tanto no pré, 
quanto no pós teste, caracterizado como não clínico. O Fator 2, Asserção (IMC=3) e o Fator 5, Cooperação com pares (IMC=3), ocorreu mudança clinicamente significativa, já que a criança pertencia, anteriormente, à intervenção, ao escore referente à população clínica, alterando para o escore referente à população não clínica após a mesma. Entretanto, para o Fator 3, Autocontrole, e para o Fator 4 Autodefesa ocorreu ausência de mudança, já que o IMC foi igual à l. Nesses fatores, a criança já pertencia, anteriormente, à intervenção, ao escore referente à população clínica e, após, continuou nessa mesma classificação.

Tabela 2. Descrição dos resultados obtidos com a professora antes e depois da intervenção.

\begin{tabular}{c|c|c|c|c|c}
\hline \multirow{2}{*}{ No $^{2}$} & Fatores & \multicolumn{2}{|c|}{ Status Clínico } & \multicolumn{2}{c}{$\begin{array}{c}\text { Indice de Mudança } \\
\text { Confiável }\end{array}$} \\
\cline { 3 - 6 } & $\mathbf{l}^{\mathbf{a}}$ Aplicação & $\mathbf{2}^{\mathbf{a}}$ Aplicação & IMC & Classificação \\
\hline 01 & $\begin{array}{c}\text { Responsabilidade e } \\
\text { Cooperação }\end{array}$ & Não clínico & Não clínico & 1 & $\begin{array}{c}\text { Ausência de } \\
\text { Mudança }\end{array}$ \\
\hline 02 & Asserção & Clínico & Não clínico & 3 & $\begin{array}{c}\text { Mudança Positiva } \\
\text { Confiável }\end{array}$ \\
\hline 03 & Autocontrole & Clínico & Clínico & 1 & $\begin{array}{c}\text { Ausência de } \\
\text { Mudança }\end{array}$ \\
\hline 04 & Autodefesa & Clínico & Não clínico & 1 & $\begin{array}{c}\text { Ausência de } \\
\text { Mudança }\end{array}$ \\
\hline 05 & $\begin{array}{c}\text { Cooperação com } \\
\text { pares }\end{array}$ & Clínico & Não clínico & 3 & $\begin{array}{c}\text { Mudança Positiva } \\
\text { Confiável }\end{array}$ \\
\hline
\end{tabular}

\section{Discussão}

Analisando o repertório social relatado por pais e professora na primeira avaliação, observa-se que, para os pais, todos os fatores foram avaliados com nível de significância clínica. Para a professora, quatro dos cinco fatores avaliados estavam nesta condição. Os resultados obtidos estão condizentes com os encontrados por Pereira (2007) e Anhão, Pfeifer e Santos (2010) que apontaram para defasagens importantes no repertório social de crianças com síndrome de Down.

$\mathrm{Na}$ avaliação com os pais, a análise dos resultados pré e pós intervenção mostraram que a intervenção com a criança obteve mudança positiva confiável, além de mudança do status clínico para não clínico em quatro dos seis fatores avaliados: Asserção Positiva, Iniciativa e Desenvoltura Social, Asserção de Enfrentamento e Civilidade. Nos fatores Cooperação e Autocontrole, ocorreu ausência de mudança, permanecendo a criança no status não clínico na segunda aplicação, pós intervenção. O fator de Asserção de Enfrentamento, um dos que melhorou com a intervenção conduzida, foi o fator observado em defasagem no repertório social de crianças e adolescentes com Síndrome de Down, nos estudos de Angélico (2004) e Anhão et al. (2010), que apontaram para a necessidade de trabalhos nesse sentido. 
$\mathrm{Na}$ avaliação com a professora, a análise dos resultados pré e pós intervenção, mostrou que a intervenção com a criança obteve mudança positiva confiável e mudança de status clínico para não clínico para dois dos cinco fatores avaliados: Asserção e Cooperação com Pares.

Comparando os resultados dos fatores Responsabilidade e Cooperação (professora) e Cooperação (pais) observou-se, no contexto escolar, como um repertório presente (não clínico) nas duas aplicações e para os pais não clínico, também nas duas aplicações. Uma hipótese é que este repertório pode ter sido sempre reforçado na escola e pouco exigido ou esperado no ambiente doméstico. Tal fato indica que é um comportamento que deve ser alvo de intervenção pontual junto aos pais.

Para o Autocontrole (pais, Fator 6; professora, Fator 3) o status permaneceu clínico nos dois contextos, nas duas avaliações, portanto, pouco sensível às intervenções realizadas. Tal resultado aponta para a necessidade de intervenção conjunta em casa e na escola. Todavia, os diferentes contextos podem ter exigências diferentes nas habilidades avaliadas, o que sugere que as intervenções programadas devem considerar necessidades específicas de cada um deles, ainda que aparentemente estejam na mesma categoria.

De maneira geral à intervenção trouxe resultados positivos para a criança, segundo a análise de mudança positiva e significância clínica. Os resultados obtidos com as intervenções ressaltam a importância de intervenções pontuais em todos os ambientes que a criança frequenta tendo em vista a melhoria do seu repertório de habilidades sociais. O estudo mostrou, também, a eficácia de uma intervenção baseada em princípios do treinamento de habilidades sociais com a criança, pais e professores.

\section{Conclusão}

O objetivo do presente estudo foi analisar os efeitos de uma intervenção em habilidades sociais com uma criança com Síndrome de Down. Os resultados permitem concluir pela eficácia dos procedimentos utilizados e, principalmente, pelo envolvimento de ações dos diversos atores: pais, professores e terapeuta em prol de um objetivo comum. Estudos como este apontam para a possibilidade de intervenções pontuais em mais de um contexto. Todavia, estudos com populações maiores devem ser feitos utilizando os mesmos instrumentos e envolvendo os múltiplos contextos de frequência do indivíduo. Uma sugestão é avaliar, também, o desempenho escolar que está diretamente associado ao repertório de habilidades sociais, o que poderia ser associado à possíveis mudanças geradas pela intervenção focalizada nas habilidades sociais. 


\section{Referências}

AGUIAR, A.A.R.; DEL PRETTE, Z.A.P.; AGUIAR, R.G.; DEL PRETTE. Método JT na Educação Especial: resultados de um programa de habilidades sociais-comunicativas com deficientes mentais. Revista de Educação Especial, Santa Maria, v. 23, n. 37, p. 241-256, 2010.

ANGÉLICO, A.P. Estudo descritivo de repertório de habilidades sociais de adolescentes com Síndrome de Down. Dissertação de Mestrado. Programa de Pós Graduação em Educação Especial. Universidade Federal de São Carlos, 2004.

ANGELICO, A.P.; DEL PRETTE, A. Avaliação do repertório de habilidades sociais de adolescentes com síndrome de Down. Psicologia: Reflexão e Crítica, v. 24, n. 2, p. 207-217, 2011.

ANHÃO, P.P.G.; PFEIFER, L.I.; SANTOS, J.L. Interação social de crianças com síndrome de down na educação infantil. Revista Brasileira de Educação Especial, Marília, v. 16, n. 1, p. 31-46, 2010.

BANDEIRA, M.; DEL PRETTE, Z.A.P.; DEL PRETTE, A.; MAGALHÃES, T. Validação das escalas de habilidades sociais, comportamentos problemáticos e competência acadêmica (SSRS-BR) no ensino fundamental. Psicologia: Teoria e Pesquisa, v. 25, n. 2, p. 271-282, 2009.

BEIDEL, D.C.; TURNER, S.M.; MORRIS, T.L. Behavioral treatment of childhood social phobia. Journal of Consulting and Clinical Psychology, v. 68, p. 1072-1080, 2000.

BELTRAME, T.S.; HERBER, V.; RIBEIRO, J. O processo de socialização de uma criança com síndrome de Down no sistema regular de ensino. Dynamis Revista Científica, v. 15, n. 2, p. 15-39, 2009.

BONOMO, L.M.M.; GARCIA, A.; ROSSETTI, C.B. O adolescente com síndrome de Down e sua rede de relacionamentos: Um estudo exploratório sobre suas amizades. Psicologia: Teoria e Pesquisa. v. 11, n. 3, p. $114-130,2009$.

DEL PRETTE, Z. A. P.; DEL PRETTE, A. Psicologia das habilidades sociais: terapia e educação. Petrópolis,: Vozes, 1999.

DEL PRETTE, Z. A. P. \& DEL PRETTE, A. Psicologia das relações interpessoais: vivências para o trabalho em grupo. Petrópolis: Vozes, 2001.

DEL PRETTE, Z. A. P.; DEL PRETTE, A. Psicologia das habilidades sociais na infância: Teoria e prática. Petrópolis: Vozes, 2005.

DEL PRETTE, Z. A. P.; DEL PRETTE, A. significância clínica e mudança confiável na avaliação de intervenções psicológicas. Psicologia: Teoria e Pesquisa, Brasília, v. 24, n. 4, p. 497-505, 2008.

FREITAS, L.C. Habilidades sociais de crianças com diferentes necessidades educacionais especiais: Comparações múltiplas. Tese de Doutorado. Programa de Pós-Graduação em Educação Especial, Universidade Federal de São Carlos, São Carlos, 2011.

GAUDERER, C. Autismo e outros atrasos do desenvolvimento: Guia prático para pais e profissionais. São Paulo: Revinter, 1997.

GRESHAM, F.M. Conceptual and definitional issues in the assessment of children's social skills: implications for classification and training. Journal of Clinical Child Psychology, v. 15, n. 1, p. 3-15, 1986.

GRESHAM, F. M. Análise do comportamento aplicada às habilidades sociais. Em Z.A.P. DEL PRETTE; A. DEL PRETTE (Eds.) Psicologia das habilidades sociais: Diversidade teórica e suas implicações. Petrópolis: Editora Vozes, 2009, p. 17-66. 
JACOBSON, N.S.; TRUAX, P. Clinical significance A statistical approach to defining meaningful change In psychotherapy reasearch. Journal of Consulting and Clinical Psychology, v. 59, p. 12-19, 1991.

JACOBSON, N.S.; TRUAX, P. A statistical approach to defining meaningful change in psychotherapy reasearch. Em A.E. KAZDIN (Ed.) Methodological issues and strategies. In clinical research. Washington Copyright.

LUCISANO, R.V.; PFEIFER, L.I.; PANUNCIO-PINTO, M.P.; ANHÃO, P.P.G.; SANTOS, J.L.F. Interação social de crianças pré-escolares com síndrome de Down. Revista do Nufen. v. 3, n. 2, p. 97-115, 2011.

MANCINI, M. C.; SILVA, P.C.; GONÇALVES, S.C.; MARTINS, S.M. Comparação do desempenho funcional de crianças portadoras de síndrome de Down e crianças com desenvolvimento normal aos 2 e 5 anos de idade. Arquivos de Neuropsiquiatria, v. 61, n. 2B, p. 409-415, 2003.

MARGISON, F.R.; BARKHAM, M.; EVANS, C.; McGRATH, G.; CLARK, J.M.; AUDEN, K.; CONNEL, J. Mensurement and psychotherapy: evidence-based practice and practice-based evidence. British Journal of Psychiatry, n. 177, p. 123-130, 2000.

OMOTE, S. Aparência e competência em Educação Especial. Temas em Educação Especial. São Carlos: UFSCar, 1990, p. 11-25.

PEREIRA, M.S. Semelhanças e diferenças de habilidades sociais entre crianças com síndrome de Down incluídas e crianças com desenvolvimento típico. Dissertação de Mestrado. Programa de Pós Graduação em Educação Especial. Universidade Federal de São Carlos, 2007.

SORESI, S; NOTA, L. A Social Skill Training for Persons with Down's Syndrome. European Psychologist, v. 5, n. 1, p. 33-43, 2000.

TEIXEIRA, F.C.; KUBO, O.M. Características das interações entre alunos com síndrome de down e seus colegas de turma no sistema regular de ensino. Revista Brasileira de Educação Especial, Marília, v. 14, n. 1, p. 75-92, 2008.

\section{Correspondência}

Olga Maria Piazentin Rolim Rodrigues - Universidade Estadual Paulista Júlio de Mesquita Filho, Faculdade de Ciências de Bauru, Departamento de Psicologia. Avenida Luiz Edmundo Carrijo Coube, sn, Vargem Limpa, CEP: 17033-360, Bauru, São Paulo, Brasil.

E-mail: olgarolim@fc.unesp.br - larihelena@yahoo.com.br - fccarlino.usp@gmail.com

Recebido em 10 de janeiro de 2013

Aprovado em 22 de maio de 2013 
